\title{
Use of the critical incident technique in primary care in the audit of deaths by suicide
}

Durham Medical Audit Advisory Group, County Durham Health Authority, Appleton House, Lanchester Road, County Durham L Redpath, audit development manager A Stacey, case discussion leader

E Holmes, chairman and general practitioner E Pugh, director of public health

Correspondence to: Ms L Redpath, Durham Medical Audit Advisory Group, County Durham Health Authority, Appleton House, Lanchester Road,
County Durham DH1 5 XZ

Accepted for publication 14 November 1996

\begin{abstract}
Objective-To explore the usefulness of the critical incident technique in primary care to improve policy and practice to prevent cases of suicide.

Design-Inviting all primary care teams in County Durham with a patient who committed suicide between 1 June 1993 and 31 May 1994 to take part in a critical incident audit with an external facilitator. Results-49 cases of suicide were available for study, registered with 31 practices. 19 (61\%) practices accounting for $27(55 \%)$ cases agreed to take part. Case discussions showed areas where practice could be improved, but no substantive preventive measures were identified within primary care, which would reduce the number of people committing suicide. The wider social and economic context was thought to be more important.

Conclusions-The use of the critical incident technique in primary care may have only a limited role in improving the management of people at risk from suicide. However, one of the potential strengths of this approach is to encourage reflection on practice in a difficult emotional area.
\end{abstract}

(Quality in Health Care 1997;6:25-28)

Keywords: critical incident technique; suicide; primary care health teams.

\section{Introduction}

Suicide is theoretically an avoidable death which accounts for $1 \%$ of deaths annually in the United Kingdom. The government, through its Department of Health, has set a national strategy for improving health which includes a target to reduce overall suicide rates by $15 \%$ by the year $2000 .^{1}$ This will require the concerted efforts of many agencies with health professionals having a major role.

This paper describes a study which uses a structured internal audit process with members of primary care teams to review deaths of their patients by suicide. To see if there is scope for primary care teams to improve policy and practice to reduce suicides, primary care team members were encouraged to recollect their relations with cases systematically and critically to allow lessons to be drawn from these cases in a constructive nonjudgemental way.

The analysis of an important event such as the death of a patient has been found to be a useful approach to audit. ${ }^{2}$ In hospitals in the
United Kingdom confidential enquiries have been used effectively as a basis for medical audit. ${ }^{34}$ These enquiries are judged by external assessors, usually by applying preset criteria.

Analysis within primary care of individual cases provides a useful educational exercise. Cases are usually randomly selected. Through clinical audit cases can be discussed in a multidisciplinary way.

The informal process of random case review can be made more structured by adapting the "critical incident" method. This was first developed by Flanagan ${ }^{5}$ in the 1950s. Participants are asked to recall situations that they think reflect good and bad practice. They then are asked to give their reasons for why they perceive practice to be good or bad. This method is known as "facilitated case discussion" and it's application to primary health care has been described by Robinson et $a l .{ }^{6}$ Through it notable or critical incidents in care, both positive and negative, are identified. The incidents may be clinical, managerial, or organisational. This method has been piloted in one inner city general practice. ${ }^{7}$

The present paper builds upon that work and uses a single diagnosis of suicide with the facilitated case discussion open to all primary care teams in County Durham with whom a patient who died from suicide was registered.

\section{Method}

\section{GATHERING DATA}

Information from coroners in County Durham relating to suicide (ICD E950-959) and open verdicts (ICD 980-989) for the study period 1 June 1993 to 31 May 1994 was obtained for residents of County Durham. For each case a record was made of age, sex, marital, and employment status, method of suicide, date, place of death, and the general practitioner named on the coroner's report.

\section{CASE DISCUSSIONS}

The general practitioner with whom the patient was registered was contacted to invite the practice to take part in the project. Participation was voluntary. It was emphasised that all members of the primary care team could attend the case discussion, even though perhaps only one or two members had had direct involvement with the case. It was left to the general practitioner to decide who should be present at the discussion.

When practices agreed to participate, the person who had the most involvement was asked to prepare a written summary of the case, which was circulated to all participants 
before the discussion. This avoided third party access to the medical records.

Meetings were set up with an external case discussion group leader (AS) who had a background in psychology and counselling and who had been recruited to the project to facilitate critical case discussions.

The content of case discussions and the types of issues arising depended on contribution of the participants. The date and time of the meeting varied according to the wishes of the practice as did the duration of the discussion, which varied from 20 to 60 minutes.

The case discussion leader outlined the project to the participants. Reassurance was given about confidentiality in every case, advice having been previously sought from one of the medical defence organisations. Each case discussion was taped and then transcribed. Tapes were erased. The case discussion leader identified critical events from the transcriptions. These included positive points arising in the discussion, matters of concern, and areas for further consideration.

All members were invited to add their observations. At the end of the meeting the facilitator summarised the discussion encouraging the team to identify areas where care could have been improved.

After the meeting individual practices received written feedback on a semistructured form outlining the main issues in the case, positive actions taken, and areas for further consideration. A date was set between two and three months later to liaise with the practice to see whether any changes had been implemented.

\section{Results}

ALL CASES

In the 12 months of the study period 42 verdicts of suicide and seven open verdicts were reported for residents of County Durham by the coroner to Durham Medical Audit Advisory Group. The male to female ratio was about $2: 1$. The average age of the men was 42 years, that for women 54 years. All cases of suicide under 30 years old $(n=6)$ were men. The most common method of suicide in men was hanging $(15(44 \%))$. The most common method for women was a drug overdose ( 8 $(53 \%))$.

Eighteen $(53 \%)$ men and $10(67 \%)$ women were unemployed or retired. Thirteen (38\%) men and three $(20 \%)$ women were recorded as being divorced, separated, or widowed.

STUDY CASES

The 49 cases reported occurred in 31 primary care teams. Of these $26 \%$ were training practices and $6 \%$ were single handed. Nineteen $(61 \%)$ of the practices in which a suicide had occurred agreed to take part in the critical incident audit. Of these $32 \%$ were training practices and $5 \%$ were single handed. This included $27(55 \%)$ of the suicides.

There were variations in the composition of team members present for the case discussions. In $10(53 \%)$ only general practitioners were present. In four of these, discussion was one to one with a single general practitioner. (The general practitioner was the one who had had most involvement with the patient.) In three (16\%) the general practitioners were accompanied by their general practice trainees. In the remaining nine meetings non-medical disciplines were represented including community psychiatric nurses at two meetings, practice nurses at three, a health promoter and practice nurses at the others.

Of all cases studied $19(70 \%)$ had seen their general practitioner within the previous month. Five $(19 \%)$ had seen their general practitioner within the previous week. Five $(19 \%)$ had not been seen in the past two years. It was reported that eight ( $29 \%)$ of the patients had made more than normal demands on the service.

In at least half of the cases patients were either receiving treatment for physical problems or had been referred for a specialist opinion on physical symptoms. In half of this group the general practitioner thought that the symptoms could be related to anxiety but were seeking a specialist opinion on physical symptoms to reassure the patient or themselves. For most of the cases of suicide the general practitioners restricted their focus of care to presenting physical problems.

Over half of the cases had a history of depression, anxiety, or neurosis. At least 11 (37\%) were taking long term antidepressants. Seven $(26 \%)$ had a history of known excessive alcohol consumption. Eight (30\%) had a previous episode of deliberate self harm or a suicide attempt. At least five (19\%) of the patients (three were schizophrenic, and two were assessed as having reactive depression) had a current diagnosed psychiatric problem.

From the discussions over half of cases had identifiable external factors which the general practitioner thought could have been relevant. These included money or relationship problems, actual or impending anniversary of a bereavement, or an impending court case. In some cases the general practitioner became aware of this information after the suicide or it had come from a source other than the patient and they did not feel able to discuss it.

Most primary healthcare teams thought it was extremely difficult to identify patients at risk of committing suicide. This was because of the perceived large number of complex factors involved. There were, however, several practices that had attempted to flag records. In the cases studied the primary healthcare team members did not think the outcome of suicide could have been averted. It was thought that wider issues in society were more important than individual intervention. For example, one practice commented that:

\begin{tabular}{l}
\hline \hline "Closing the pits, the lack of \\
money, and lack of jobs has much \\
more effect on the suicide rate. \\
than anything we can do."
\end{tabular}


The role of the community psychiatric nurse was often mentioned. There was a diversity of views about the role and expectations of community psychiatric nurses. This ranged from some general practitioners experiencing a very close working relationship, to other general practitioners not knowing what they could reasonably expect from the service. Some practices had a community psychiatric nurse attached. Others claimed they did not know how to access the services of the community psychiatric nurse for cases they considered appropriate. There were repeated comments that there was not always agreement between general practitioners and community psychiatric nurses about what constituted an appropriate or priority referral. There was a perception that the focus on reducing waiting lists meant that community psychiatric nurses were less involved in crisis intervention, which was where they were most needed in the opinion of the general practitioners.

There was evidence to suggest the need for an improvement in communications between secondary and primary care. For example, situations were described when patients had been treated in hospital without the general practitioner knowing. There were some occasions when general practitioners had referred patients to hospital for specialist advice which they thought had not been forthcoming.

The process of notification of death was often mentioned. It was perceived that there was not a system in place for ensuring that a general practitioner was notified of a patient's death by suicide. General practitioners often found out from the local informal networks, including other patients. This was thought to be unsatisfactory as it resulted in a delay or absence of the general practitioner offering help to the patient's family. Overall little specific help was identified for the bereaved carers.

General practitioners experienced difficulty in their own clinical practice in identifying emotional rather than physical problems. Some thought that they did not have the skill. Most said that they did not have the time. When general practitioners suspected that the underlying problem was emotional they would generally carry out routine investigations to attempt to treat physical symptoms. Almost all general practitioners stated that they would ask and check whether someone had suicidal feelings. Most general practitioners did not see it as their role to provide counselling services. One general practitioner commented:

"If I think they are depressed I always ask them if they are feeling suicidal. If they don't tell me what can I do? I haven't the time to spend with them talking about their feelings."

EXAMPLES OF GOOD PRACTICE, POSITIVE CRITICAL EVENTS IDENTIFIED

These included:

- Routine bereavement visits

- Follow up work with families

- The use of approved counsellors
- Family folders

- The use of case summaries in notes

- Positive working relations with other organisations.

All practices that took part in case discussions were offered a review visit three months later to discuss changes implemented. Examples of changes included:

- Improved liaison and access to the community psychiatric nurse service instigated by the general practitioner

- Practice based counselling service established

- Tightening up of number and type of antidepressants available in the community.

\section{Discussion}

The characteristics of suicide cases reflected the national picture with twice as many men as women committing suicide. Of the men many were young, a noticeable proportion being under $45 .^{\circ}$ In line with other studies most patients had consulted their general practitioner within the previous month. ${ }^{9}$

To our knowledge this is the first study to use a critical incident technique with primary care teams to explore issues surrounding cases of suicides. Although the outcome of facilitated case discussion was varied, areas were identified where improvements to care could be made and also areas of good practice were identified which may merit wider dissemination.

The value of this method depends on the active participation of members of primary healthcare teams. The fact that $12(39 \%)$ eligible practices turned down the invitation to take part highlights the reluctance of some practices to take part in this type of voluntary exercise despite the study being conducted by the Medical Audit Advisory Group in a manner ensuring anonymity. Concern was expressed in a few cases on issues of confidentiality and worries of litigation. This has been recognised. ${ }^{6}$ This reinforces the need for protocols protecting confidentiality and avoiding litigation to be adhered to, to maintain the confidence of general practitioners and ensure voluntary participation.

One of the potential strengths of this method is to encourage multidisciplinary audit reflecting the multidisciplinary teamwork usual in primary care. Leaving the decision about attendance to the general practitioner with whom the patient was registered resulted in most meetings having general practitioners only. In only two meetings were community psychiatric nurses present. Clearly, participating practices will have to be encouraged to send more of their members to the meetings to ensure multidisciplinary involvement.

From the discussions primary care teams failed to identify any substantial deficiencies for which they were responsible and which may have averted a case of suicide. There are several possible explanations. Firstly, it may be that for such a sensitive issue as suicide the critical incident technique may be unacceptable. This was not the case in the pilot study of deaths 
conducted in a single practice where areas for improvement were made.

Secondly, the technique may be appropriate but the way it was carried out in this study was not conducive to self or team criticism. For example, some teams may not be used to meeting regularly and reviewing patient care. Analysis may be threatening to team members particularly when an external facilitator is used. This may occur despite the facilitator encouraging openness and stressing the exercise to be non-judgemental. Perhaps different group leadership skills might be more effective. It is recognised that external facilitators could be threatening and could affect existing team dynamics. ${ }^{6}$

The impotence of primary care to influence suicide seems surprising given the fact that a substantial proportion of patients who committed suicide had clinical risk factors such as a previous episode of deliberate self harm or a current psychiatric problem including schizophrenia. The difficulty in identifying patients at high risk as opposed to having clinical risk factors was highlighted. Overall the wider social and economic context was perceived to be of more importance. Despite the primary care team feeling powerless at preventing suicides examples of good practice were identified.

The need for general practitioners to understand the role of and means to appropriately access the community psychiatric nursing service shows the need for primary care practices to work closely on an equal footing with other disciplines to use all the resources and skills available. The community psychiatric nurse may have a wider role in supporting general practitioners in their care of patients with mental health risk factors, particularly those who are depressed or who have schizophrenia.

At the time of writing review meetings were ongoing. The results of this study have led to the formation of a multidisciplinary suicide task force, which was launched in January 1996. The work of this group has to a large extent been exploratory and has brought together people and organisations that had no previous relationships to consider the issues of prevention of suicide within County Durham.

\section{Conclusions}

Athough the use of the critical incident technique in primary care may have only a limited role in improving the management of people at risk from suicide, examples of good practice were identified and one of the potential strengths of this approach is to encourage multidisciplinary reflection. The wider economic and social environment is perceived to be of greater importance than interventions by primary healthcare teams.

We thank the Durham primary healthcare teams who have taken part in the project and have welcomed us into their practices to discuss such a difficult area of practice. We also thank Rosie Stacey, John Spencer, and Louise Robinson from the Repartment of Primary Care at the Newcastle Medical School, for their help and support in establishing the predical School, project. We than edge the support of coroners in County Durham without which the project could not have taken place.

1 Department of Health. The health of the nation. A strategy for health in England. London: HMSO, 1992.

2 Buckley G. Clinically significant event. In: Marinker M, ed. Medical audit in general practice. London: MSD Foundation $1990144-167$.

3 Department of Health. Report on confidential enquiries into maternal mortality 1982-4. London: HMSO, DoH, 1989.

4 Campling EA, Devlin HB, Lunn JN. Report of the national confidential enquiry into perioperative deaths 1989 . London: confidential enquiry

5 Flanagan JC. The critical incident technique. Psychol Bull 1954;51:327-58

6 Robinson LA, Stacey R, Spencer JA, Bhopal RS. How to use facilitated case discussions for significant event auditing. BMF 1995;311:315-9.

7 Berlin A, Spencer JA, Bhopal RS, van Zwanenberg TD Audit of deaths in general practice: pilot study of the critical incident technique. Quality in Health Care 1992;1:2315.

8 Hawton K. By their own young hand. BMF 1992;304:1000

9 Vassilas CA, Morgan HG. GPs contact with victims of suicide. $B M \mathcal{F}$ 1993;307:300-1 